\title{
ON THE DOMAIN OF REGULARITY OF GENERALIZED AXIALLY SYMMETRIC POTENTIALS
}

\author{
PETER HENRICI
}

Introduction. It is known $[3 ; 7]$ that for $2 \nu \neq 0,-1,-2, \cdots$ every solution $u(x, y)$ of the partial differential equation ${ }^{1}$

$$
\frac{\partial^{2} u}{\partial x^{2}}+\frac{\partial^{2} u}{\partial y^{2}}+\frac{2 \nu}{y} \frac{\partial u}{\partial y}=0,
$$

which depends analytically on the two real variables $x$ and $y$ in a domain containing a segment of the singular line $y=0$, is uniquely determined by its values on $y=0$. In this note we shall consider the following problem: If the function $g(z)=u(z, 0)$ is continued to complex values of $z$, to what extent does its domain of regularity determine the domain of regularity of $u(x, y)$ ?

A partial answer to this has been given by Erdélyi [1], who proved that if $g(z)$ is holomorphic in a $y$-convex domain ${ }^{2} \mathfrak{y}$, the function $u(x, y)$ is regular at all real points $(x, y)$ for which $x+i y \in \mathfrak{y}$. The general results of I. N. Vekua in the theory of elliptic differential equations with analytic coefficients ${ }^{3}$ suggest the following wider (and perhaps more natural) statement:

THEOREM I. Let $g(z)$ be holomorphic in a simply connected domain $\mathfrak{y}$, and let $2 \nu \neq 0,-1,-2, \cdots$. Then there exists a unique solution $u(x, y)$ of (1) satisfying $u(z, 0)=g(z)$ for $z \in \mathfrak{y} ; u(x, y)$ is an analytic function of its arguments in the domain $D(\mathfrak{y})$ of all complex points $(x, y)$ for which $x+i y \in \mathfrak{y}, x-i y \in \mathfrak{y}$.

For $\operatorname{Re} \nu>0$ and convex domains $y$ Theorem $I$ is contained in an otherwise more general result of ours. ${ }^{4}$ If $x$ and $y$ are restricted to real values and if $y$ is $y$-convex, the theorem reduces to Erdélyi's result. ${ }^{5}$

Proof of Theorem I. Since uniqueness in the small, as established in [3], implies uniqueness in the large by analytic continuation, it suffices to show the existence of a function with the desired properties.

Received by the editors January 10, 1956 and, in revised form, March 5, 1956.

${ }^{1}$ Frequently called equation of generalized axially symmetric potentials.

$2 \mathrm{By}$ this is meant a domain which contains with the point $x+i y$ also the points $x+i y t,-1 \leqq t \leqq 1$.

${ }^{3}$ See e.g. $[6, \S 9]$.

4 See $[2$, Satz III,].

5 For a discussion of the role played by the exceptional values $2 \nu=0,-1,-2, \ldots$ see [4]. 
We first assume $\operatorname{Re} \nu>0$. If $\mathfrak{y}$ is convex, the function

$$
u(x, y)=\frac{\Gamma(\nu+1 / 2)}{\Gamma(\nu) \Gamma(1 / 2)} \int_{-1}^{1} g(x+i y t)\left(1-t^{2}\right)^{\nu-1} d t,
$$

where the integration is along the real axis, meets the conditions of the theorem (see [2, proof of Satz $\mathrm{III}_{\nu}$ ). In the nonconvex case the difficulty arises that no longer one and the same path of integration can be used for all points $P=(x, y) \in D(y)$. We therefore integrate along a curvilinear path which depends continuously on $P$. This path $C(P)$ may be constructed in the following manner: Let $\phi$ be a differentiable 1-1 mapping of $\mathfrak{y}$ on a convex domain $\mathfrak{y}^{\prime}$, and let $P^{\prime}=\left(x^{\prime}, y^{\prime}\right)$ where $x^{\prime} \pm i y^{\prime}=\phi(x \pm i y)$. Let $\zeta_{P}$ denote the mapping of the $t$-plane on the $T$-plane given by $T=x+i y t$. This mapping depends analytically on the parameter $P$ and so does its inverse, except for $y=0$. We now put

$$
C(P)= \begin{cases}\zeta_{P}^{-1} \phi^{-1} \zeta_{P^{\prime}}(S), & y \neq 0, \\ S, & y=0,\end{cases}
$$

where $S$ denotes the segment $-1 \leqq t \leqq 1$. The path $C(P)$ depends continuously on $P$ (also at $y=0$ ) and has the property that $x+i y t \in \mathfrak{y}$ for $t \in C(P)$ and $P \in D(\mathfrak{y})$. Every point $P_{0} \in D(\mathfrak{y})$ possesses a neighborhood $N$ such that $\zeta_{P}\left(C\left(P_{0}\right)\right) \in \mathfrak{y}$ for $P \in N$. We may therefore replace $C(P)$ by $C\left(P_{0}\right)$ for $P \in N$ and conclude by a classical theorem that (2) is an analytic function of $P$ at $P=P_{0}$. The remaining assertions of Theorem I can be verified as in [2].

For $\operatorname{Re} \nu \leqq 0$ the path $C(P)$ has to be replaced by a figure eight loop as used by Mackie [4]. The idea of the proof remains the same.

Remarks. 1 . Theorem I sets up a mapping $u=\theta[g]$ of the class of all functions $g(z)$ regular in $\mathfrak{y}$ into the class of all solutions $u(x, y)$ of $(1)$ regular in $D(\mathfrak{y})$. For $\operatorname{Re} \nu>0$ and for special domains $y$ we can show that this mapping is onto (and thus 1-1); indeed, it is onto a seemingly less restricted class of solutions. This is implied by

THEOREM II. Let $\operatorname{Re} \nu>0$, and let the solution $u(x, y)$ be real-analytic in a simply connected domain $y$ which is symmetric with respect to $y=0$. Then the function $g(x)=u(x, 0)$ can be continued analytically to a function $g(z)$ which is holomorphic for $z \in \mathfrak{y}$.

For convex domains this theorem is a special case of Satz $I_{v}$ in [2]. The proof given there is also valid for nonconvex domains.

Szegö $[\mathbf{5}]$ has recently proved a theorem which in slightly extended 
form can be stated as follows: Let $g(z)$ be regular in $|z|<r$, and let $u(x, y)=\theta[g]$, where $\nu=1 / 2$. Then, for any real $\vartheta,(x, y)=(r \cos \vartheta$, $r \sin \vartheta)$ is a regular point of $u(x, y)$ if and only if $z=r e^{i \vartheta}$ and $z=r e^{-i \vartheta}$ are regular points of $g(z)$. This is clearly a corollary of the logical product of the Theorems I and II for convex regions $\mathfrak{y}$. This case, however, was already covered in our earlier paper [2].

2. If the function $\phi$ used in the proof of Theorem I is sufficiently smooth, the length of $C(P)$ depends continuously on $P$. It follows that the mapping $\theta$ is continuous in the following sense:

THEOREM III. For every $\epsilon>0$ and every closed subdomain $y$ of $y$ there exists $a \delta>0$ such that $|\theta[g(z)]|<\epsilon$ in $D(\bar{y})$ if $|g(z)|<\delta$ for $z \in \bar{y}$.

It follows by Runge's theorem that every $u(x, y)$ satisfying the hypothesis of Theorem II can be approximated uniformly in every $D(\overline{\mathfrak{y}})$ by linear combinations of the functions $\theta\left[z^{n}\right], n=0,1,2, \cdots$. These functions can be expressed explicitly in terms of ultraspherical polynomials (see [2]).

3. We finally mention that the considerations of this note can be extended with little change to solutions of the equation obtained from (1) by adding a term $+k^{2} u$ ( $k$ a complex constant) to the left. The role of (2) is then taken over by

$$
u(x, y)=\frac{\Gamma(\nu+1 / 2)}{\Gamma(1 / 2)} \int_{-1}^{1} g(x+i y t)(k y / 2)^{-\nu+1} J_{\nu-1}\left(k y\left(1-t^{2}\right)^{1 / 2}\right) d t,
$$

where $J_{\nu-1}$ is the Bessel function of order $\nu-1$.

\section{REFERENCES}

1. A. Erdélyi, Singularities of generalized axially symmetric potentials, Comm. Pure Appl. Math. vol. 9 (1956) pp. 403-414.

2. P. Henrici, Zur Funktionentheorie der Wellengleichung, Comment. Math. Helv. vol. 27 (1953) pp. 235-293.

3. M. A. Hyman, Concerning analytic solutions of the generalized potential equation, Neder. Akad. Wetensch. Ser. A vol. 57 (1954) pp. 408-413.

4. A. G. Mackie, Contour integral solutions of a class of differential equations, Journal of Rational Mechanics and Analysis vol. 4 (1955) pp. 733-750.

5. G. Szegö, On the singularities of zonal harmonic expansions, Journal of Rational Mechanics and Analysis vol. 3 (1954) pp. 561-564.

6. I. N. Vekua, Novye metody resenija elliptičeskikh uravnenij, OGIZ, Moscow and Leningrad, 1948.

7. A. Weinstein, Discontinuous integrals and generalized potential theory, Trans. Amer. Math. Soc. vol. 63 (1948) pp. 342-354.

AMERICAN UNIVERSITY ${ }^{6}$

B The author is now at the University of California, Los Angeles. 